COMPETING EFFECTS DESIGNS AND MODELS FOR TWO-DIMENSIONAL

FIELD ARRANGEMENTS

\author{
by \\ Walter T. Federer ${ }^{1}$ and Kaye E. Basford ${ }^{2}$ \\ ${ }^{1}$ Biometrics Unit, Cornell University, Ithaca, NY 14853, U.S.A. \\ ${ }^{2}$ Department of Agriculture, University of Queensland, St. Lucia, \\ Queensland 4067, Australia
}




\title{
COMPETING EFFECTS DESIGNS AND MODELS FOR TWO-DIMENSIONAL FIELD ARRANGEMENTS
}

\author{
Walter T. Federer ${ }^{1}$ and Kaye E. Basford ${ }^{2}$ \\ ${ }^{1}$ Biometrics Unit, Cornell University, Ithaca, NY 14853, U.S.A. \\ ${ }^{2}$ Department of Agriculture, University of Queensland, St. Lucia, \\ Queensland 4067, Australia
}

\section{SUMMARY}

Three methods of constructing balanced nearest neighbor row-column or competition effect designs are presented. Statistical response models for two-dimensional layouts and competing effects are formulated and corresponding statistical analyses are developed for designs of this type. The problem of obtaining solutions for all competing effects is discussed and illustrated. Two numerical examples illustrating aspects of design and statistical analyses are presented; one is an actual experiment and the other is artificial to demonstrate effect of competition on estimates of parameters. The problems of appropriate borders, spatial arrangements, measuring and/or eliminating competition effects, and the consequence of not being able to obtain estimable contrasts among the competition effects are discussed.

\section{Introduction}

Various spatial statistics can take account of correlation between adjoining experimental units (plots) in field experiments. Nearest neighbor and competition effects designs and analyses for onedimensional layouts were considered in papers edited by Kempton (1984). Also, Kempton (1982), Kempton and Lockwood (1984), and Besag and Kempton (1986), among others, considered statistical analyses for competition effects. Kempton (1982) proposed a single degree of freedom for competition effects which is a diagnostic statistic similar to Tukey's one-degree-of-freedom for nonadditivity. The present paper considers designs and statistical analyses for assessing the competition effect of individual treatments in two-dimensional layouts of field experiments. Three methods of constructing

Key words: Latin square; Repeated measures; F-square; Non-orthogonal analyses; Eigenvalue; Eigenvector; Contrasts; Border; Nearest neighbor; Spatial statistics; Competition; Field trials. 
row-column designs for $\mathbf{v}$ treatments are given. The designs are balanced for competition effects. They are also balanced for nearest neighbor analysis but that is not the topic of this paper. Two numerical examples illustrate various aspects of design and statistical analysis.

\section{Construction of Two-Dimensional Designs}

Various row-column designs have been constructed which take account of the number and position of neighbors. Most were constructed for plant breeding experiments where, for the purposes of pollination, a cultivar is bordered by all its neighbors. A historical account and description of these may be found in Freeman (1979a, 1979b, 1981). He also showed similarities and differences between various designs and illustrated how to construct some square and rectangular nearest neighbor designs. Further row-column designs constructed in the repeated measures context (a non-directional nearest neighbor in the Freeman sense) were given by Hedayat and Federer (1984).

Three methods for constructing experiment designs that are balanced for competition effects are given below. Latin square designs balanced for one-period carry-over or residual effects (see, e.g., Williams, 1949, and Bradley, 1958) may be used to construct latin square designs balanced for competition effects. Such latin squares were denoted as row complete in Dénes and Keedwell (1974). They denote a latin square design which is balanced for residual effects in both rows and columns as a complete latin square; in such squares, a treatment will occur equally often next to each of the other treatments but will not appear next to itself. The design is said to be balanced for competition effects. The idea of balance may be used in any two-dimensional layout and not solely for row-column designs.

Construction Method I. Utilizing the results in Sections 2.3 and 3.1 of Dénes and Keedwell (1974), row-column designs may be constructed that are balanced for competition effects of adjacent plots. Complete latin squares may be constructed as follows. Let $\mathrm{v}=2 \mathrm{~m}$, for $\mathrm{m}$ any positive integer, and rearrange the rows of a cyclic latin square of order $\mathrm{v}$ to have the first element of the rows as

a) $0,1,2 \mathrm{~m}-1,2,2 \mathrm{~m}-2,3,2 \mathrm{~m}-3, \cdots, \mathrm{m}+1, \mathrm{~m}$, or as

b) $0,2 \mathrm{~m}-1,1,2 \mathrm{~m}-2,2,2 \mathrm{~m}-3,3, \cdots, \mathrm{m}-1, \mathrm{~m}$. 
A latin square in either the a) or b) arrangement is balanced for residual effects or is row complete. Rearranging the columns by either a) or b) results in a complete latin square balanced for competition effects of a treatment with the other treatments. Other methods for constructing complete latin squares may be found in Freeman (1979b). These designs are also nearest-neighbor designs and are denoted as $\operatorname{NND}(\mathrm{v}, \mathrm{v} ; 0,4)$ where 0 is the number of times a treatment borders itself and 4 is the number of times a treatment is adjacent to each of the other treatments. Note that two-dimensional nearest neighbor designs may be rectangular, and then a balanced nearest neighbor design is denoted as $\mathrm{NND}(\mathrm{w}, \mathrm{x}, \mathrm{v} ; \mathrm{y}, \mathrm{z})$ which has $\mathrm{w}$ rows, $\mathrm{x}$ columns, $\mathbf{v}$ treatments, $\mathrm{y}$ adjacencies of a treatment with itself, and $\mathrm{z}$ adjacencies of two different treatments.

To illustrate Construction Method I and procedure b) on both rows and columns for $v=4$,

\begin{tabular}{|c|c|c|c|c|}
\hline $\mathrm{LS}(4)$ & & $\mathrm{RM}(4)$ & & $\mathrm{NN}(4 ; 4 ; 0,4)$ \\
\hline$A B C D$ & & $\mathrm{ABCD}$ & & $\mathrm{ADBC}$ \\
\hline BCDA & $\rightarrow$ & DABC & $\rightarrow$ & DCAB \\
\hline CDAB & & BCDA & & BACD \\
\hline DABC & & CDAB & & CBDA \\
\hline
\end{tabular}

An extra row on the above design with the last row repeated produces NNDs with $\mathrm{v}+1$ rows and $\mathrm{v}$ columns for $\mathrm{v}$ treatments and every treatment is bordered by every other treatment including itself.

Construction Method II. Construct an F-square FS $\left(2 \mathrm{v} ; 2^{\mathrm{v}}\right)$ (see Hedayat and Seiden, 1970) as the Kronecker product of a $\mathrm{J}$ matrix (all ones) of side 2 and a cyclic latin square of order $\mathrm{v}$, any integer, i.e., $\mathrm{J}_{2} \otimes \mathrm{LS}(\mathrm{v})$ where $\otimes$ denotes Kronecker product. Then apply the procedure of Construction Method I to this F-square. The designs obtained by this method are denoted as $\operatorname{NND}(2 \mathrm{v}, \mathrm{v} ; 8,16)$ designs where $2 \mathrm{v}$ refers to the order of the $\mathrm{F}$-square, $\mathrm{v}$ is the number of treatments, 8 is the number of times a treatment appears next to itself, and 16 is the number of times a given treatment is bordered by a treatment other than itself. Instead of $\mathrm{J}_{2}$, the matrices $\mathrm{J}_{3}, \mathrm{~J}_{4}$, etc. could also be used to develop other F-squares.

For $v=3$ and using procedure $b$ ) of $I$ on an F-square of order $2 \mathrm{v}$ results in 


$$
\begin{aligned}
& \mathrm{F}(6 ; 2,2,2) \quad \mathrm{ABC} A \mathrm{ABC} \quad \mathrm{ABC} \mathrm{ABC} \quad \text { ACB BCA } \\
& \text { BCA BCA CAB CAB CBA ABC } \\
& \left(\begin{array}{ll}
1 & 1 \\
1 & 1
\end{array}\right) \otimes\left[\begin{array}{l}
\mathrm{ABC} \\
\mathrm{BCA} \\
\mathrm{CAB}
\end{array}\right] \rightarrow \begin{array}{l}
\mathrm{CAB} \text { CAB } \\
\mathrm{ABC} \text { ABC } \\
\mathrm{BCA} \text { BCA } \\
\mathrm{CAB} \text { CAB }
\end{array} \rightarrow \begin{array}{l}
\text { BCA BCA } \\
\text { BCA BCA } \\
\text { CAB CAB } \\
\text { ABC ABC }
\end{array} \rightarrow \begin{array}{l}
\text { BAC CAB } \\
\text { CAC CAB ABC } \\
\text { ACB BCA }
\end{array}=\operatorname{NDD}(6,3 ; 8,16),
\end{aligned}
$$

where $\otimes$ denotes a symbolic Kronecker product. The last plan above is a nearest neighbor design for three treatments in an F-square of order 6 . Treatment $i, i \neq i$, appears next to treatment $i, 16$ times and treatment i borders itself 8 times.

Construction Method III. If a cultivar is to border itself for Construction Method I designs, simply repeat the last row of the design, as Patterson and Lucas (1959) did for repeated measures designs. Each of the $v$ treatments would then appear $v+1$ times in the design. The treatments would remain orthogonal to rows but would be in a balanced block arrangement (see Shafiq and Federer, 1979) with respect to columns.

Any row-column design may be used as a nearest neighbor design. However, not all contrasts among a set of effects may be estimable. For most designs, the Kempton (1982) single degree of freedom for competition should be estimable even if solutions for all competition effects may not be obtainable. We describe some of the effects of not being able to estimate all competition effects in Example 2.

\section{Response Model Equations for Block and Row-Column Designs}

For a model taking into account competing effects of four neighbors in a row-column design, consider the following equation for the response from the plot in row $f$ and column $g$ whose treatment is $\mathrm{h}$ :

$$
\mathrm{Y}_{\mathrm{fghijkm}}=\mu+\rho_{\mathrm{f}}+\gamma_{\mathrm{g}}+\tau_{\mathrm{h}}+\alpha_{\mathrm{i}}+\alpha_{\mathrm{j}}+\alpha_{\mathrm{k}}+\alpha_{\mathrm{m}}+\epsilon_{\mathrm{fghijkm}}
$$

where $\mu$ is a general mean effect, $\rho_{\mathrm{f}}$ is the effect of row $\mathbf{f}(\mathrm{f}=1, \cdots, \mathrm{r}), \gamma_{\mathrm{g}}$ is the effect of column $\mathrm{g}$ $(\mathrm{g}=1, \cdots, \mathrm{c}), \tau_{\mathrm{h}}$ is the effect of treatment $\mathrm{h}(\mathrm{h}=1, \cdots, \mathrm{v}), \alpha_{\mathrm{p}}$ is the competition effect of treatment $\mathrm{p}=1, \cdots, \mathrm{v}$ in four adjacent positions $(\mathrm{i}, \mathrm{j}, \mathrm{k}, \mathrm{m}=1, \cdots, \mathrm{v}, \mathrm{x})$, where $\mathbf{x}$ is an outside border for the row-column design, and $\epsilon_{\text {fghijkm }}$ are random error effects distributed with mean zero and common error variance 
$\sigma_{\epsilon}^{2}$. The competition effect of any treatment is independent of the position occupied by the treatment. For a blocked design where each plot has four neighbors, simply delete $\rho_{\mathrm{f}}$ and $\mathrm{f}$ from the above equation and let $\gamma_{\mathrm{g}}$ denote the block effect. Also, if each plot has only two neighbors, delete $\alpha_{\mathrm{k}}, \alpha_{\mathrm{m}}$, $k$, and $m$ from (1) to obtain the response model equation.

Diagonal neighbors are not considered because, for rectangular plots, they touch plot fgh on a corner only, and hence can cause little or no competition with it. Also, model (1) is most plausible when plots are square. When plots are rectangular with dimensions of length $\ell$ and width $w$, the weights $4 \ell /(2 \ell+2 w)$ might reasonably be given to the two $\alpha_{\mathrm{p}}$ on the longer sides of the plot and weights $4 \mathrm{w} /(2 \ell+2 \mathrm{w})$ on the shorter sides. If different spacings are used, replace $\ell$ by $\ell+\mathrm{d}_{1}$ and $\mathrm{w}$ by $w+d_{2}$, where $d_{1}$ and $d_{2}$ are spacing widths between plots.

In row-column designs a solution for $\alpha_{\mathrm{X}}$, the competition effect of the border $\mathrm{x}$, is not possible since $\alpha_{\mathrm{X}}$ is completely confounded with the first and last rows and the first and last columns. Despite this it may be useful to leave $\alpha_{\mathbf{X}}$ in (1) in order to understand which estimable contrasts are affected by an outside border effect. We omit $\alpha_{\mathbf{X}}$ in our solutions and numerical examples. In order to make the effect of a border equal to zero, a composite of equal amounts of all the treatments in an experiment may perhaps be used as the border $\mathrm{x}$. The solutions for competition effects add to zero and hence a border with all competition effects equally represented should not exhibit a competition effect. The use of a single cultivar $\mathrm{x}$ could exhibit a nonestimable competition effect on all border plots.

For the complete latin squares obtained from Construction Method I, solutions for row, column, treatment, and competition effects subject to the usual constraints are possible when $\mathrm{v} \geq 8$. From Construction Method II, solutions exist for $v \geq 4$. For $v=6$, none of the six complete squares given by Freeman (1979b) results in solutions for all effects. Solutions are obtainable when $v=4$ or 6 only if a row (column) complete latin square or one from Construction Method III is used; the design will not be balanced for competition effects.

Instead of explicit solutions for the row-columns designs obtained by the three construction methods, the following general solution is used because of the numerous designs involved and of the wide availability of PC computer software such as GAUSS, GENSTAT and SAS. This procedure 
handles all situations including unbalanced designs and missing observations. Using the standard linear model notation, for equation (1), let the design matrix be X. Subtracting the matrix

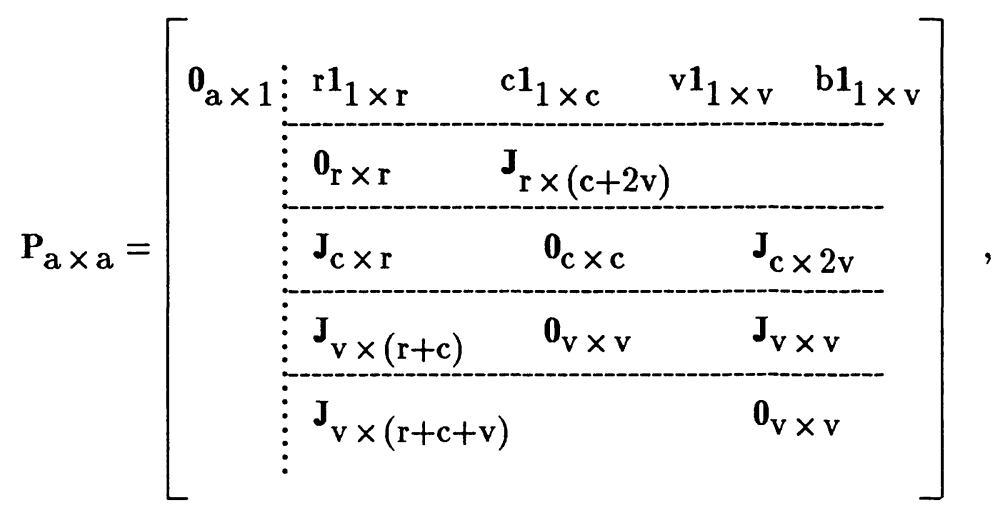

for $\mathrm{a}=\mathrm{r}+\mathrm{c}+2 \mathrm{v}+1$ from $\mathbf{X}, \mathbf{X}$, we obtain $\mathbf{Z}=\mathbf{X}, \mathbf{X}-\mathbf{P}$ which will have an inverse when solutions for all effects are possible. For $b=r+c+v+1$, let

$$
\begin{aligned}
\mathbf{Z}_{\mathrm{a} \times \mathrm{a}} & =\left[\begin{array}{cc}
\mathbf{D}_{\mathrm{b} \times \mathrm{b}} & \mathbf{A}_{\mathrm{b} \times \mathrm{v}} \\
\mathbf{N}_{\mathrm{v} \times \mathrm{b}} & \mathbf{F}_{\mathrm{v} \times \mathrm{v}}
\end{array}\right], \\
\mathbf{X}, \mathbf{Y} & =\left[\begin{array}{c}
\mathbf{W}_{1, \mathrm{~b} \times 1} \\
\mathbf{W}_{2, \mathrm{v} \times 1}
\end{array}\right], \quad \text { and } \\
\hat{\boldsymbol{\beta}} & =\mathbf{Z}^{-1} \mathbf{X}, \mathbf{Y} .
\end{aligned}
$$

The last equation above produces solutions for the $1+r+c+2 v$ effects when $\mathbf{Z}$ has an inverse. The solutions for $\hat{\boldsymbol{\alpha}}$ may also be obtained as

$$
\hat{\boldsymbol{\alpha}}=\left(\mathbf{F}-\mathbf{N D}^{-1} \mathbf{A}\right)^{-1}\left(\mathbf{W}_{2}-\mathbf{N D}^{-1} \mathbf{W}_{1}\right) .
$$

The sum of squares for the competition effects eliminating all else may be computed as

$$
\hat{\alpha}_{\iota}\left(\mathbf{W}_{2}-\mathbf{N D}^{-1} \mathbf{W}_{1}\right) \text {. }
$$

Likewise, the treatment effects and sums of squares for treatments eliminating all other effects may be computed as follows. Rearrange $\mathbf{Z}$ by interchanging the columns for treatment effects and the columns for competition effects. Then, interchange the rows for treatment effects and those for competition effects. The resulting matrix may be represented as 


$$
\mathbf{Z}^{+}=\left[\begin{array}{cc}
\mathbf{E}_{\mathrm{b} \times \mathrm{b}} & \mathbf{G}_{\mathrm{b} \times \mathrm{v}} \\
\mathbf{H}_{\mathrm{v} \times \mathrm{b}} & \mathbf{K}_{\mathrm{v} \times \mathrm{v}}
\end{array}\right]
$$

also partition the $\mathbf{X}, \mathbf{Y}$ vector as $\left[\begin{array}{l}\mathbf{W}_{3} \\ \mathbf{W}_{4}\end{array}\right]$. Then a solution for treatment effects is

$$
\hat{\tau}=\left(\mathbf{K}-\mathbf{H} \mathbf{E}^{-1} \mathbf{G}\right)^{-1}\left(\mathbf{W}_{4}-\mathbf{H} \mathbf{E}^{-1} \mathbf{W}_{3}\right) \text {. }
$$

The corresponding sum of squares is computed as

$$
\hat{\tau}_{\prime}\left(\mathbf{W}_{4}-\mathbf{H E}^{-1} \mathbf{W}_{3}\right) \text {. }
$$

\section{Illustrative Examples}

We now present examples illustrating the computational procedure. Example 1 demonstrates estimation of competition effects for a latin square of order 6, with allowance for a rectangular experimental unit. Here the treatment sum of squares eliminating all else is much reduced from the treatment ignoring competition effects sum of squares. The variances for contrasts are described. Example 2 illustrates the method of analysis for designs constructed by Method II. The data are artificial and residuals have been included. Thus, the sum of squares for the "error" line in the ANOVA is known, i.e., the sum of the squared known residuals. This design by Construction Method II for $v=3$ does not allow solutions for all competition effects. The biasing effect of not being able to estimate all competition effects is illustrated.

Example 1 Das and Giri (1979), page 77, gave the following field layout and data for a wheat

\begin{tabular}{|c|c|c|c|c|c|c|c|}
\hline Row & 1 & 2 & 3 & 4 & 5 & 6 & Total \\
\hline 1 & $219 \mathrm{~F}$ & $250 \mathrm{E}$ & $227 \mathrm{D}$ & $162 \mathrm{C}$ & $182 \mathrm{~B}$ & $91 \mathrm{~A}$ & 1131 \\
\hline 2 & $227 \mathrm{E}$ & $141 \mathrm{C}$ & $91 \mathrm{~A}$ & $191 \mathrm{D}$ & $213 \mathrm{~F}$ & $195 \mathrm{~B}$ & 1058 \\
\hline 3 & $204 \mathrm{~B}$ & $91 \mathrm{~A}$ & $225 \mathrm{~F}$ & $229 \mathrm{E}$ & $250 \mathrm{D}$ & $207 \mathrm{C}$ & 1206 \\
\hline 4 & $77 \mathrm{~A}$ & $204 \mathrm{~B}$ & $240 \mathrm{E}$ & $199 \mathrm{~F}$ & $182 \mathrm{C}$ & $250 \mathrm{D}$ & 1152 \\
\hline 5 & $250 \mathrm{D}$ & $231 \mathrm{~F}$ & $209 \mathrm{C}$ & $204 \mathrm{~B}$ & $91 \mathrm{~A}$ & $227 \mathrm{E}$ & 1212 \\
\hline 6 & $152 \mathrm{C}$ & $186 \mathrm{D}$ & $191 \mathrm{~B}$ & $77 \mathrm{~A}$ & $230 \mathrm{E}$ & $198 \mathrm{~F}$ & 1034 \\
\hline Total & 1129 & 1103 & 1183 & 1062 & 1148 & 1168 & 6793 \\
\hline
\end{tabular}
experiment in a latin square design of order six:

Column (yields in grams/10) 
The treatment totals are: $\mathrm{Y} \ldots \mathrm{A} \cdot=518, \mathrm{Y} \ldots \mathrm{B} \cdot=1180, \mathrm{Y} \ldots \mathrm{C} \cdot=1053, \mathrm{Y} \ldots \mathrm{D} \cdot=1354, \mathrm{Y} \ldots \mathrm{E} \cdot$ $=1403, \mathrm{Y}_{\ldots \mathrm{F}} .=1285$. The competition effect totals are: $\mathrm{Y} \ldots \mathrm{A}=4006.82, \mathrm{Y} \ldots \mathrm{B}=2899.90$, $\mathrm{Y} \ldots \mathrm{C}=4087.28, \mathrm{Y} \ldots \mathrm{D}=3796.82, \mathrm{Y} \ldots \mathrm{E}=3675.44, \mathrm{Y} \ldots \mathrm{F}=4130.22$. The solutions for the effects are:

$$
\begin{array}{llll}
\hat{\mu}=188.694 & & & \\
\hat{\rho}_{1}=-1.063 & \hat{\gamma}_{1}=-0.452 & \hat{\tau}_{\mathrm{A}}=-102.262 & \hat{\alpha}_{\mathrm{A}}=-5.947 \\
\hat{\rho}_{2}=-12.478 & \hat{\gamma}_{2}=-4.750 & \hat{\tau}_{\mathrm{B}}=14.985 & \hat{\alpha}_{\mathrm{B}}=-2.039 \\
\hat{\rho}_{3}=11.696 & \hat{\gamma}_{3}=8.497 & \hat{\tau}_{\mathrm{C}}=-16.397 & \hat{\alpha}_{\mathrm{C}}=0.074 \\
\hat{\rho}_{4}=2.425 & \hat{\gamma}_{4}=-11.831 & \hat{\tau}_{\mathrm{D}}=36.423 & \hat{\alpha}_{\mathrm{D}}=3.106 \\
\hat{\rho}_{5}=14.784 & \hat{\gamma}_{5}=2.630 & \hat{\tau}_{\mathrm{E}}=43.355 & \hat{\alpha}_{\mathrm{E}}=1.662 \\
\hat{\rho}_{6}=-15.363 & \hat{\gamma}_{6}=5.907 & \hat{\tau}_{\mathrm{F}}=23.898 & \hat{\alpha}_{\mathrm{F}}=3.145
\end{array}
$$

The treatments are: $A=$ no nitrogen $(\mathrm{N}), \mathrm{B}=40 \mathrm{~kg} \mathrm{~N} /$ hectare, $\mathrm{C}=80 \mathrm{~kg} /$ hectare, $\mathrm{D}=120$ $\mathrm{kg} /$ hectare, $\mathrm{E}=160 \mathrm{~kg} /$ hectare, $\mathrm{F}=200 \mathrm{~kg} /$ hectare. The plot size was $8 \times 0.6$ meters.

The total perimeter of a plot was $2(8+0.6)=17.2$ meters. Therefore the coefficients for competition effects in the design matrix are $4(8 / 17.2)=1.86$ and $4(0.6 / 17.2)=0.14$. That is, the sides of the experimental unit are bordered by a neighbor for a length of 8 meters, whereas the ends are bordered by only 0.6 meters. The design matrix is $\mathbf{X}=\left[\begin{array}{ll}\mathbf{X}_{1} & \mathbf{X}_{2}\end{array}\right]$ where $\mathbf{X}_{1}$ is the usual design matrix for a latin square of order six and

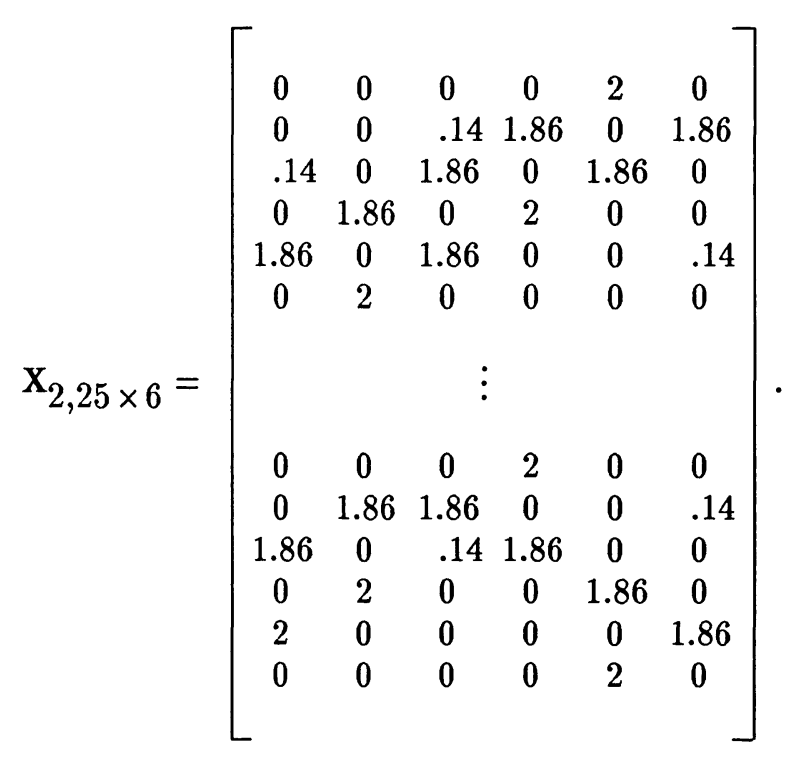


An ANOVA for this example is:

\begin{tabular}{|c|c|c|c|}
\hline Source of variation & $\mathrm{df}$ & Sum of squares & Mean square \\
\hline Total & 36 & $1,381,069$ & - \\
\hline Correction for mean & 1 & $1,281,801.36$ & - \\
\hline Row (ignoring competition) & 5 & $4,559.47$ & 911.89 \\
\hline Column (ignoring competition) & 5 & $1,650.47$ & 330.09 \\
\hline Nitrogen levels (ignoring competition) & 5 & $88,612.47$ & - \\
\hline Remainder & 20 & $4,445.22$ & 222.26 \\
\hline Competition (eliminating all else) & 5 & $1,102.68$ & 220.54 \\
\hline Error & 15 & $3,342.54$ & 222.84 \\
\hline Nitrogen (eliminating all else) & 5 & $53,114.90$ & $10,622.98$ \\
\hline
\end{tabular}

The sum of squares for competition effects eliminating all other effects is computed as:

$$
\hat{\boldsymbol{\alpha}}_{\prime}\left(\mathbf{W}_{2}-\mathrm{ND}^{-1} \mathbf{W}_{1}\right)=\left[\begin{array}{r}
-5.949 \\
-2.039 \\
0.074 \\
3.106 \\
1.663 \\
3.145
\end{array}\right]^{\prime} \cdot\left[\begin{array}{r}
-108.946 \\
-19.792 \\
11.978 \\
89.478 \\
-33.456 \\
60.738
\end{array}\right]=1,102.68 .
$$

The sum of squares for treatments eliminating all other effects is computed as:

$$
\hat{\tau}_{1}\left(\mathbf{W}_{4}-\mathbf{H E}^{-1} \mathbf{W}_{3}\right)=\left[\begin{array}{r}
-102.262 \\
14.986 \\
-16.398 \\
36.422 \\
43.355 \\
23.896
\end{array}\right] \cdot\left[\begin{array}{r}
-373.601 \\
-41.860 \\
-29.990 \\
111.456 \\
154.406 \\
179.590
\end{array}\right]=53,114.90 .
$$

The estimated variance-covariance matrix for $\hat{\boldsymbol{\alpha}}$ is ${ }^{1}$

$$
\hat{\sigma}_{\epsilon}^{2}\left(\mathbf{F}-\mathbf{N D}^{-1} \mathbf{A}\right)^{-1}=222.84 \quad\left[\begin{array}{rrrrrr}
0.058 & -0.001 & 0.001 & -0.002 & -0.023 & -0.004 \\
-0.001 & 0.053 & -0.006 & 0.000 & -0.000 & -0.017 \\
0.001 & -0.006 & 0.054 & -0.020 & -0.013 & 0.013 \\
-0.002 & 0.000 & -0.020 & 0.050 & 0.015 & -0.014 \\
-0.023 & -0.000 & -0.013 & 0.015 & 0.054 & -0.003 \\
-0.004 & -0.017 & 0.013 & -0.014 & -0.003 & 0.055
\end{array}\right] \text {. }
$$

The estimated variance-covariance matrix for $\hat{\tau}$ is ${ }^{1}$

\footnotetext{
$\overline{1}$ Various computer programs give different variance-covariance matrices. However, adding an appropriate constant (of the order of .01) times a J matrix (all ones) to any one of them yields another. The variance of a contrast is identical for all forms obtained. We obtained three different forms.
} 


$$
\hat{\sigma}_{\epsilon}^{2}\left(\mathbf{K}-\mathbf{H E}^{-1} \mathbf{G}\right)^{-1}=222.84 \quad\left[\begin{array}{rrrrrr}
0.275 & -0.065 & 0.027 & -0.015 & -0.037 & 0.034 \\
-0.065 & 0.283 & -0.023 & 0.019 & 0.040 & -0.036 \\
0.027 & -0.023 & 0.235 & -0.052 & 0.001 & 0.029 \\
-0.015 & 0.019 & -0.052 & 0.251 & 0.023 & -0.008 \\
-0.037 & 0.040 & 0.001 & 0.023 & 0.231 & -0.039 \\
0.034 & -0.036 & 0.029 & -0.008 & -0.039 & 0.239
\end{array}\right] .
$$

Something appears to be amiss with the data for this example. Only two values, 77 and 91 , were obtained for treatment A, 9 of the 36 values are integral multiples of one pound, 15 of the 36 are multiples of one-half pound, and if the remaining ones should be multiples of one-fourth pound, some arithmetic errors were made in converting to grams. Also, it appears that the residual sum of squares may be too large, perhaps because of nonadditivity, heterogeneous variances for treatments, or because gradients run diagonally through the square. However, either the contrast A versus the rest or a linear trend of effects on nitrogen level would account for a large proportion of the treatment (eliminating all other effects) and competition (eliminating all other effects) sums of squares. These sums of squares, respectively, are $45,846.17$ and 777.74 for the first contrast. These were computed as $\ell \hat{\alpha} \ell\left(\mathbf{W}_{2}-\mathbf{N D}^{-1} \mathbf{W}_{1}\right) / \ell \ell$; where $\ell=\left(\begin{array}{llllll}-5 & 1 & 1 & 1 & 1 & 1\end{array}\right)$. Even though the "Error" sum of squares is probably too large, the single degree of freedom sum of squares for competition effects is significant at about the $8 \%$ level. Biologically, this would be explainable by the fact that when nitrogen was available, plants got off to an earlier start and were larger. The larger plants made use of the nutrients in the adjoining plots with smaller plants. A linear trend, i.e., $\boldsymbol{\ell}=\left(\begin{array}{llllll}-5 & -3 & -1 & 1 & 3 & 5\end{array}\right)$, gave very similar sums of squares, namely $38,394.11$ and 753.55 . The remainder mean square for nitrogen effects after removal of the contrast A versus rest sum of squares was 1817.18 with four degrees of freedom, resulting in an F-value of 8.2. A linear regression among the nonzero levels of nitrogen would account for a large proportion of this variation.

Some items to note are:

i) The sum of squares for treatment eliminating all other effects is only $3 / 5$ as large as the treatment ignoring competition effects sum of squares.

ii) Plot shape needs to be considered in assessing competition effects. 
iii) A linear regression of treatment and competition effects on amount of nitrogen applied would account for a considerable and significant proportion of their respective sums of squares.

iv) The inferences for fertilizer effect would be little affected with regard to slope of fertilizer treatment responses to increasing nitrogen even if competition were ignored.

v) The design matrix $\mathbf{X}$ has coefficients other than 0 and 1 and some are not integers.

vi) The variances for competition contrasts are much smaller than for fertilizer treatment contrasts. The extra replication for competition effects accounts for this.

Example 2 An artificial example is constructed from the following values for the effects for a design from Construction Method II for $v=3$ treatments and response model equation (1):

$$
\begin{array}{rlll}
\mu=20 & & & \\
\rho_{1}=-5 & \gamma_{1}=-3 & \tau_{\mathrm{A}}=-7 & \epsilon_{12 \mathrm{C}}=-1 \\
\rho_{1}=5 & \gamma_{2}=-3 & \tau_{\mathrm{B}}=2 & \epsilon_{13 \mathrm{~B}}=1 \\
\rho_{3}=0 & \gamma_{3}=6 & \tau_{\mathrm{C}}=5 & \epsilon_{21 \mathrm{C}}=1 \\
\rho_{4}=0 & \gamma_{4}=0 & \alpha_{\mathrm{A}}=-3 & \epsilon_{23 \mathrm{~A}}=-1 \\
\rho_{5}=0 & \gamma_{5}=0 & \alpha_{\mathrm{B}}=-1 & \epsilon_{31 \mathrm{~B}}=-1 \\
\rho_{6}=0 & \gamma_{6}=0 & \alpha_{\mathrm{C}}=4 & \epsilon_{32 \mathrm{~A}}=1
\end{array}
$$

\begin{tabular}{|c|c|c|c|c|c|c|c|}
\hline \multirow[b]{2}{*}{ Rows } & \multicolumn{6}{|c|}{ Columns (yields and treatments) } & \multirow[b]{2}{*}{ Total } \\
\hline & 1 & 2 & 3 & 4 & 5 & 6 & \\
\hline 1 & $13 \mathrm{~A}$ & $11 \mathrm{C}$ & $24 \mathrm{~B}$ & 17B & $15 \mathrm{C}$ & $16 \mathrm{~A}$ & 96 \\
\hline 2 & $23 \mathrm{C}$ & $26 \mathrm{~B}$ & $22 \mathrm{~A}$ & $17 \mathrm{~A}$ & $29 \mathrm{~B}$ & $25 \mathrm{C}$ & 142 \\
\hline 3 & $18 \mathrm{~B}$ & $10 \mathrm{~A}$ & $33 \mathrm{C}$ & $27 \mathrm{C}$ & $12 \mathrm{~A}$ & $22 \mathrm{~B}$ & 122 \\
\hline 4 & $19 \mathrm{~B}$ & $9 \mathrm{~A}$ & $33 \mathrm{C}$ & $27 \mathrm{C}$ & $12 \mathrm{~A}$ & $22 \mathrm{~B}$ & 122 \\
\hline 5 & $17 \mathrm{C}$ & $21 \mathrm{~B}$ & $18 \mathrm{~A}$ & $12 \mathrm{~A}$ & $24 \mathrm{~B}$ & $20 \mathrm{C}$ & 112 \\
\hline 6 & $18 \mathrm{~A}$ & $17 \mathrm{C}$ & $28 \mathrm{~B}$ & $22 \mathrm{~B}$ & $20 \mathrm{C}$ & $21 \mathrm{~A}$ & 126 \\
\hline Total & 108 & 94 & 158 & 122 & 112 & 126 & 720 \\
\hline
\end{tabular}

All other $\epsilon_{\text {fghijkm}}$, or residuals, are set equal to zero. The sum of residuals is zero over rows, columns, treatments, and competition effects. From the above values the data and design are:

For this design not all effects have solutions with the constraints $\Sigma \hat{\rho}_{\mathrm{f}}=\Sigma \hat{\gamma}_{\mathrm{g}}=\Sigma \hat{\tau}_{\mathrm{h}}=\Sigma \hat{\alpha}_{\mathrm{i}}=0$. To determine which linear contrasts of the $\hat{\boldsymbol{\alpha}}$ are not estimable, one may obtain the eigenvalues and 
eigenvectors for the matrix $\left(\mathbf{F}-\mathbf{N D}^{-1} \mathbf{A}\right)$. Or, one can go back to the original $\mathbf{X}, \mathbf{X}$ matrix, and apply the constraints for the row, column, and treatment effects but not for the competition effects. Let the matrix $\mathbf{X}, \mathbf{X}$ with the above constraints be

$$
\mathbf{Z}_{\mathrm{a} \times \mathrm{a}}^{*}=\left[\begin{array}{cc}
\mathbf{D}_{\mathrm{b} \times \mathrm{b}} & \mathbf{A}_{\mathrm{b} \times \mathrm{v}}^{*} \\
\mathbf{N}_{\mathrm{v} \times \mathrm{v}}^{*} & \mathbf{F}_{\mathrm{v} \times \mathrm{v}}
\end{array}\right],
$$

where $\mathbf{D}$ and $\mathbf{F}$ have been defined previously and $\mathbf{A}^{*}$ and $\mathbf{N}^{*}$ are the corresponding submatrices of $X, X$. In this form the eigenvalues and eigenvectors of $\left(\mathbf{F}-\mathbf{N}^{*} \mathbf{D}^{-1} \mathbf{A}^{*}\right)$ can be obtained and examined. Omitting the last constraint but using the first three on $\mathbf{X}, \mathbf{X}$, denoting the resulting matrix as $\mathbf{Z}^{*}$ as in equation (10), the following matrix is obtained:

$$
\mathbf{F}-\mathbf{N}^{*} \mathbf{D}^{-1} \mathbf{A}^{*}=\left[\begin{array}{rrr}
0.8889 & -4.4444 & 3.5556 \\
-4.4444 & 22.2222 & -17.7778 \\
3.5556 & -17.7778 & 14.2222
\end{array}\right]
$$

where $\mathbf{Z}^{*}=\left[\begin{array}{cc}\mathbf{D} & \mathbf{A}^{*} \\ \mathbf{N}^{*} & \mathbf{F}\end{array}\right]$. The nonzero eigenvalue of the above matrix is $37 \frac{1}{3}$ and the corresponding eigenvector is $\left(\begin{array}{lll}0.15430 & -0.77152 & 0.61721\end{array}\right)=\mathrm{E}$. Since only one eigenvalue is nonzero, the total sum of squares for competition effects is obtained from the single degree of freedom contrast $0.15430 \alpha_{A}-$ $0.77152 \alpha_{B}+0.61721 \alpha_{C}=Q_{1}$. Now form the matrix $\mathbf{Z}_{1}=\left[\begin{array}{cc}\mathbf{D} & \mathbf{A}^{*} \mathbf{E} \\ \mathbf{E} \mathbf{N}^{*} & \mathbf{E} \mathbf{F E}\end{array}\right]$ where $\mathbf{D}_{16 \times 16}$ has the following vector as its first column and main right diagonal:

$$
\left(\begin{array}{llllllllllllllll}
36 & 6 & 6 & 6 & 6 & 6 & 6 & 6 & 6 & 6 & 6 & 6 & 6 & 12 & 12 & 12
\end{array}\right) \text {, }
$$

with all other elements zero.

$$
\begin{aligned}
& \left(\mathbf{A}^{*} \mathbf{E}\right)_{1 \times 16}^{\prime}=\left(\mathbf{E}^{\prime} \mathbf{N}^{*}\right)_{1 \times 16}= \\
& {\left[\begin{array}{rrrrrrrrrr}
-0.0004 & -0.3087 & -1.2345 & 1.5430 & 1.5430 & -1.2345 & -0.3087 & -0.3087 & -1.2345 & 1.5430 \\
1.5430 & -1.2345 & -0.3087 & -1.2346 & 6.1720 & -4.9378 & & & &
\end{array}\right]}
\end{aligned}
$$

and $\left(\mathbf{E}^{\prime} \mathbf{F E}\right)_{1 \times 1}=45.3333$. The matrix $\mathbf{Z}_{1}$ now has an inverse. Form totals $\mathbf{T}=\mathbf{X}^{\prime} \mathbf{Y}$ where $\mathbf{X}$ is the 
incidence matrix and $\mathbf{Y}$ is the observation vector. Let $\mathbf{T}_{1}$ consist of the first 16 totals from $\mathbf{T}$ followed by $\mathbf{E}^{\prime}$ times the last three totals of $\mathbf{T}$. Then, the solutions for effects are obtained as $\hat{\beta}_{1}=\left(\mathbf{Z}_{1}\right)^{-1} \mathbf{T}_{1}$ and are:

\begin{tabular}{cccc} 
Solution & Bias & Solution & Bias \\
\hline$\hat{\mu}=20.000$ & 0 & $\hat{\gamma}_{1}=-1.857$ & $-3(8 / 21)$ \\
$\hat{\rho}_{1}=-3.857$ & $-3(8 / 21)$ & $\hat{\gamma}_{2}=-3.762$ & $2(8 / 21)$ \\
$\hat{\rho}_{2}=4.238$ & $2(8 / 21)$ & $\hat{\gamma}_{3}=5.619$ & $8 / 21$ \\
$\hat{\rho}_{3}=-0.381$ & $8 / 21$ & $\hat{\gamma}_{4}=-0.381$ & $8 / 21$ \\
$\hat{\rho}_{4}=-0.381$ & $8 / 21$ & $\hat{\gamma}_{5}=-0.762$ & $2(8 / 21)$ \\
$\hat{\rho}_{5}=-0.762$ & $2(8 / 21)$ & $\hat{\gamma}_{6}=1.143$ & $-3(8 / 21)$ \\
$\hat{\rho}_{6}=1.143$ & $-3(8 / 21)$ & $\hat{\tau}_{\mathrm{A}}=-4.714$ & $-6(8 / 21)$ \\
& & $\hat{\tau}_{\mathrm{B}}=1.238$ & $2(8 / 21)$ \\
& & $\hat{\tau}_{\mathrm{C}}=3.476$ & $4(8 / 21)$ \\
& & $\hat{\alpha}_{1}=2.777$ & 0
\end{tabular}

Adding the bias to each of the solutions results in the effect values used to construct the example. The bias is a multiple of $8 / 21$. Because this particular design did not allow solutions for $\hat{\alpha}_{\mathrm{A}}$, $\hat{\alpha}_{\mathrm{B}}$, and $\hat{\alpha}_{\mathrm{C}}$, the other parameter estimates are biased.

To obtain the sum of squares $(1 \mathrm{df})$ for competition effects eliminating all other effects, proceed as before except that $\mathbf{Z}_{1}$ and $\mathbf{T}_{1}$ are used in place of $\mathbf{Z}$ and $\mathbf{X}^{\prime} \mathbf{Y}$.

The following ANOVA table was obtained using these solutions and the methods described above and in previous examples:

\begin{tabular}{|c|c|c|}
\hline Source of variation & $\mathrm{df}$ & Sum of squares \\
\hline Total & 36 & 15,734 \\
\hline Correction for mean & 1 & 14,400 \\
\hline Row (ignoring competition) & 5 & 194.67 \\
\hline Column (ignoring competition) & 5 & 394.67 \\
\hline Treatment (ignoring competition) & 2 & 450.66 \\
\hline Remainder (ignoring competition) & 23 & 294.00 \\
\hline Competition (eliminating all else) & 1 & 288.00 \\
\hline Residual & 22 & 6.00 \\
\hline Treatment (eliminating all else) & 2 & 429.77 \\
\hline
\end{tabular}

Some items to note are:

i) This design does not allow solutions for all effects. 
ii) The solutions obtained for the effects are biased.

iii) The solutions obtained ignoring competition effects would also have been biased. They are:

$$
\begin{array}{lll}
\hat{\mu}=20 & & \\
\hat{\rho}_{1}=-4 & \hat{\gamma}_{1}=-2 & \hat{\tau}_{\mathrm{A}}=-5 \\
\hat{\rho}_{2}=11 / 3 & \hat{\gamma}_{2}=-13 / 3 & \hat{\tau}_{\mathrm{B}}=8 / 3 \\
\hat{\rho}_{3}=1 / 3 & \hat{\gamma}_{3}=19 / 3 & \hat{\tau}_{\mathrm{C}}=7 / 3 \\
\hat{\rho}_{4}=1 / 3 & \hat{\gamma}_{4}=1 / 3 & \\
\hat{\rho}_{5}=-4 / 3 & \hat{\gamma}_{5}=-4 / 3 & \\
\hat{\rho}_{6}=1 & \hat{\gamma}_{6}=1 &
\end{array}
$$

iv) The estimated error variance for an F-square would have been $294 / 23$ and would be $6 / 22$ in the above ANOVA whereas it should have been $6 / 21$ as the example was constructed.

v) The above illustrates that if competition effects are present, they should all be estimable as they were in Example 1. Otherwise, the results may be vitiated by biased estimates of the parameters.

vi) All F-tests are biased.

vii) If a different arrangement of columns had been used, solutions for all competition effects may have been possible. 


\section{Discussion}

Competition can be important in field experiments. The first author has seen experiments in maize wherein one cultivar was completely eliminated by its neighbors and in sugarcane where a neighboring cultivar had a very visible effect on the first two rows of a cultivar even though the rows were ten feet apart. Several other examples have been seen. Unless an experimenter desires to measure competitive effects (see, e.g., Jensen and Federer, 1964, 1965), an arrangement of plots is desirable which would eliminate competition. This can be achieved through spacing or through using border rows. The latter utilizes additional space and material which are often limiting. Also, additional spacing may increase the heterogeneity within blocks, resulting in larger error mean squares. As repeatedly advocated by the late Dr. LeRoy Powers, geneticist and plant breeder, the use of space can eliminate the adverse effects of competition from adjoining plots and missing plants within a plot. In genetic studies, competitive effects must be eliminated from genetic effects whereas in commercial field arrangements intra-cultivar competitive effects must be considered in evaluating cultivars for sole cropping conditions. Likewise, inter-cultivar competitive effects must be considered for intercropping mixtures of cultivars. Experimenters have confused the goal of experiments and still do so. The conditions of an experiment must emulate conditions to be used in practice in order to make meaningful inferences. In sole-cropping practices, inter-cultivar competition is not a factor; if it is present but ignored in an experiment evaluating cultivars, the inferences may be meaningless.

To effectively eliminate inter-plot competition, we suggest that the rows between plots be approximately twice the distance of lateral root growth. For cereals, this is approximately the height of the plants. To obtain the same density per hectare, Dr. Powers recommended that the density within plots be increased to satisfy this requirement. Although the spatial arrangement was different but the density was the same as used in practice, Dr. Powers found that differences between yields of cultivars were of the same order of magnitude in experiments and in farmers' fields. Another spatial arrangement for maize cultivars would be to have a plot of two rows, 0.25 meters apart and with 1.75 meters between pairs of rows. This arrangement would be comparable in density to one where rows are one meter apart and would effectively eliminate competition between plots for most maize 
cultivars. (Lamberts (1983), e.g., used the paired row arrangement of maize in several experiments on sweet corn.) Other spatial arrangements are given in Federer (1990, Chapter 9) for sole and intercropping experiments.

For border rows in an experiment, we suggest a composite of equal amounts of all treatments. If the competition effects sum to zero, this composite would exert zero effect on the plots on the outsides of an experiment. However, competition effects need not sum to zero (see Federer, 1990, Chapters 6 and 7). As competition effects are estimated in the statistical analyses presented, they do sum to zero. Hence, a composite may be the answer for borders for some experiments, whether a varietal trial or a fertilizer, spraying, or other type of experiment. If the treatments are dates of planting, for example, an average date of planting could be used for the borders or the outside plots could be divided into equal areas with each area having one of the treatments.

The designs obtained by construction methods I, II, and III may be used not only for plant pollination studies as mentioned earlier, but also for plant association and plant competition studies. A plan obtained by Construction Method II has been used to study association and competition among five plant species in Australia.

\section{ACKNOWLEDGEMENT}

The authors thank the referees and an Associate Editor for their valuable comments on many aspects of this paper. These were used to obtain a much better form for the paper. 


\section{REFERENCES}

Besag, J. and Kempton, R. A. (1986). Statistical analysis of field experiments using neighbouring plots. Biometrics 42, 231-251.

Bradley, J. V. (1958). Complete counterbalancing of immediate sequential effects in a Latin Square design. Journal of the American Statistical Association 53, 525-528.

Das, M. N. and Giri, N. C. (1979). Design and Analysis of Experiments. New York: Hallsted Press, John Wiley and Sons, Inc.

Dénes, J. and Keedwell, A. D. (1974). Latin Squares and Their Applications. New York and London: Academic Press.

Federer, W. T. (1990). The Design and Analysis of Intercropping Experiments. (In press.)

Freeman, G. H. (1979a). Some two-dimensional designs balanced for nearest neighbours. Journal of the Royal Statistical Society, Series B 41, 88-95.

Freeman, G. H. (1979b). Complete latin squares and related designs. Journal of the Royal Statistical Society, Series B 41, 253-262.

Freeman, G. H. (1981). Further results on quasi-complete latin squares. Journal of the Royal Statistical Society, Series B 43, 314-320.

Hedayat, A. S. and Federer, W. T. (1984). Orthogonal F-rectangles for all even v. Calcutta Statistical Association Bulletin 33, 85-92.

Hedayat, A. A. and Seiden, E. (1970). F-square and orthogonal F-square designs: A generalization of Latin square and orthogonal Latin square design. Annals of Mathematical Statistics 41, 20352044.

Jensen, N. F. and Federer, W. T. (1964). Adjacent row competition in wheat. Crop Science 4, 641645.

Jensen, N. F. and Federer, W. T. (1965). Competing ability in wheat. Crop Science 5, 449-452.

Kempton, R. A. (1982). Adjustment for competition between varieties in plant breeding trials. Journal of Agricultural Science 98, 599-611.

Kempton, R. A., editor (1984). Spatial Methods in Field Experiments. Biometric Society Workshop, 
13th December 1984, University of Durham, Plant Breeding Institute, Cambridge, England CB2 2LQ.

Kempton, R. A. and Lockwood, G. (1984). Inter-plot competition in variety trials of field beans (Vicia faba L.). Journal of Agricultural Science 103, 293-302.

Lamberts, M. L. (1983). Relay intercropping peas and sweet corn - A study of experimentation on intercropping. Ph.D. Thesis, Cornell University, Ithaca, NY, May.

Patterson, H. D. and Lucas, H. L. (1959). Extra-period changeover designs. Biometrics 15, 116-132.

Shafiq, M. and Federer, W. T. (1979). Generalized N-ary balanced block designs. Biometrika 66, $115-123$.

Williams, E. J. (1949). Experimental designs balanced for the estimation of residual effects of treatments. Australian Journal of Scientific Research 2(A), 149-168. 